\title{
Autoeficacia en padres de dependientes a opiáceos
}

\author{
Araque F. (1); López-Torrecillas F. ${ }^{(2)}$; De los Riscos Ma(3); Godoy J. F. \\ (1) Doctor en Psicología. Director del Centro Provincial de Drogodependencias de Jaén. (2) Doctora en Psicología. Prof. asociada Departamento de Personalidad, \\ Evaluación y Tratamiento Psicológico. Facultad de Psicología. Universidad de Granada. (3) Master en Drogodependencias. Centro Provincial de Drogodepen- \\ dencias de Jaén. (4) Catedrático de psicología. Director del Departamento de Personalidad, Evaluación y Tratamiento Psicológico. \\ Facultad de Psicología. Universidad de Granada.
}

Enviar correspondencia a:

Francisco Araque. Centro Provincial de Drogodependencias. Recinto Hospitalario Princesa de España. Carretera de Madrid S/N. 23009 Jaén

\section{RESUMEN}

El presente trabajo investiga niveles en autoeficacia de padres de personas dependientes a opiáceos. Se han utilizado dos grupos, uno constituido por padres de sujetos en programas de mantenimiento con metadona y otro por padres de sujetos en programas libres de drogas. Se han utilizado como medidas la Escala de Autoeficacia de Bäbler y la escala de Autoeficacia de Sherer y Adams. Los resultados señalan mayor puntuación en autoeficacia de los padres en el grupo libre de drogas y mayor puntuación en autoeficacia de las madres frente a sus maridos en ambos grupos. Estos resultados apuntan la necesidad de planificar estrategias terapéuticas dirigidas a incrementar la autoeficacia de los padres de sujetos en tratamiento para su drogodependencia (tanto programas libres de drogas como en mantenimiento con opiáceos). Así mismo, se considera que estos resultados podrían ser utilizados en la planificación de actividades preventivas en el marco de las escuelas de padres.

Palabras Clave: autoeficacia, heroinómanos, opiáceos, factores familiares, padres.

\section{SUMMARY}

The present work investigates levels of self-efficacy in parents of opiate dependents. There were two groups, one comprising parents of users in maintenance programmes with metadone, and another for parents of users in drug-free programmes. They were evaluated using the Bäbler Self-efficacy Scale, and the Sherer and Adams Self-efficacy Scale. The results show higher parents self-efficacy scores in the drug-free group and higher self-efficacy scores in the mothers in comparison with their husbands in both groups. These results show the necessity of planning therapeutic strategies directed at increasing the self-efficacy of the parents of users in treatment (in both drug-free programmes or in maintenance with opiates). In addition, it is considered that these results could be used in planning preventive activities within the framework of teaching parents.

Key words: self-efficacy, addiction, drug users, opiates, family factors, parents

\section{INTRODUCCIÓN}

$\mathbf{L}$ a teoría de la Autoeficacia ha generado un gran número de investigaciones en el terreno de la psicología en general y en el de las drogodependencias en particular. Originariamente Bandura (1977) distingue entre expectativas de resultados y expectativas de eficacia personal. Las expectativas de resultados son las creencias por parte de un sujeto de que determinado comportamiento producirá un determinado resultado y las expectativas de eficacia personal son las creencias de que uno es capaz de ejecutar exitosamente el comportamiento requerido para obtener unos determinados resultados. Posteriormente estos conceptos son manejados por Bandura $(1986,1999)$ de forma alternativa e intercambiable como bien se expone en los trabajos desarrollados por Villamarin
(1990, 1994). Así, ambos tipos de expectativas contribuirían a la ejecución de la conducta y a su efectividad siendo en muchas ocasiones las expectativas de resultados sustituidas por las expectativas de eficacia. Las expectativas de eficacia estarían referidas a la elección de actividades, el esfuerzo, la persistencia en actividades, patrones de pensamiento y respuestas emocionales (Villamarín, 1994). Las expectativas de autoeficacia son cogniciones específicas y cambiantes y se modifican a partir de nuevas informaciones sobre las capacidades de los individuos. El modelo de cambio psicológico que propone esta teoría se basa en la modificación de la autoeficacia a través de los logros, la experiencia vicaria, la persuasión verbal y la activación emocional.

La teoría de la Autoeficacia se ha aplicado con éxito a las distintas áreas relacionadas con la salud 
(Bandura, 1999). Así, la autoeficacia influye en dos niveles; en un primer nivel, la confianza en manejar estresores activa los sistemas biológicos que median en la salud y la enfermedad, en un segundo nivel se relaciona con variables modificables implicadas en la salud (Bandura, 1992, 1999). En este sentido, se ha relacionado la autoeficacia con en el manejo del estrés (Lazarus y Folkman, 1987), con el funcionamiento del sistema inmunológico (Wiendelfeld et al., 1990), con el manejo del dolor (Litt, 1988) y con las variaciones en la presión y ritmo sanguíneo (Bandura et al., 1988).

En el campo de las adicciones y la prevención de recaída, desde la teoría de la Autoeficacia se ha desarrollado un modelo teórico que está siendo muy utilizado (Marlatt y Gordon, 1985; Bandura, 1992; Marlatt, 1993). La autoeficacia percibida representa la confianza de que uno es capaz de modificar las conductas arriesgadas para la salud. Así, se ha correlacionado tanto con la aparición de la conducta adictiva como con la facilitación de los procesos de cese. En el primero la autoeficacia se refiere a la habilidad autopercibida para afrontar y resistir a la presión de consumir drogas. En este sentido, se ha demostrado que la baja autoeficacia predice tanto las intenciones como la conducta de consumo de alcohol y tabaco en adolescentes (Ellickson y Hays, 1991). En el segundo, una vez iniciado el consumo de sustancias se trata de eliminar el consumo abusivo y así, reducir el daño (Marlatt et al., 1999). En este sentido, Condiotte y Lichtenstein (1981) y Diclemente (1981) han observado la relación entre autoeficacia y el descenso de la tasa de tentaciones y recaídas en el tabaquismo.

Ha sido precisamente en el campo del tabaquismo donde más se ha estudiado la teoría de la Autoeficacia desde los programas de deshabituación (Diclemente, 1981; Cooney et al., 1982; Becoña et al., 1989). En esta misma línea, Limoneo et al. (1991) han conectado los resultados de programas de deshabituación tabáquica con expectativas de resultado y autoeficacia.

En alcoholismo, Annis y Dennis (1990) han señalado que la autoeficacia mejora los programas de deshabituación. Por su parte, Aasd et al. (1995) han unido las expectativas de autoeficacia y resultado a las investigaciones epidemiológicas de carácter preventivo. De igual manera, se ha comprobado la relación entre autoeficacia y consumo controlado de alcohol (Sithartan y Kavanaugh, 1990) y consecuencias de la abstinencia (Marlatt et al., 1995).

Con los opiáceos, Grossop et al. (1990) han relacionado los resultados de los tratamientos con los niveles de autoeficacia. A nivel global, Villamarín (1994) en su revisión señala que las expectativas ejercen un papel modulador del efecto terapéutico de los tratamientos psicológicos y constituyen un buen predictor de conductas.
La percepción de autoeficacia también se ha investigado en el sistema familiar. En este sentido, se ha señalado que los progenitores que poseen un nivel de autoeficacia elevado favorecen las competencias de los hijos (Teti y Gelfand, 1991; Rudkin et al., 1992). En esta línea Cutrona y Troutman (1986) y Oliof y Aboud (1991) señalan que la autoeficacia parental ejerce función protectora contra la tensión emocional y el abatimiento de los hijos. Con respecto a la estructura familiar, Parish y Newman (1990) encuentran puntuaciones más altas en autoeficacia en los hijos únicos y en los primeros. Los resultados son contradictorios en el caso del tamaño de la familia (Schneewind, 1999). En el campo de la interacción familiar, Schneewind (1999) en su revisión señala fuertes relaciones entre autoeficacia y clima familiar y entre autoeficacia y modelado en adolescentes.

Otra variables de interés en relación a la familia el campo de las drogodependencias han sido señaladas por diferentes autores (Stanton y Tood, 1988; Charro y Martínez, 1995; Ayerbe et al., 1997; Colleti, 1998). Destacamos aquellos que investigan supervisión parental/tipo de disciplina (Jurich y Polson,1985; Kandel, 1990; Tarter et al., 1993), apego/cercanía (Kandel, 1992; Jurich y Polson, 1985; Johnson y Pandina, 1991; Barnea et al.,1992), dependencia química multigeneracional (Alameda et al., 1987; Rodríguez y Sanz, 1987), comunicación familiar/cohesión (Kornblit y Mendes, 1990; Cancrini, 1991), autonomía/dependencia (Stanton y Tood, 1988; Navarro y Prieto, 1992; Ripple y Luthar, 1996; Coletti, 1998), conflicto (Otero et al., 1989; Kornblit et al., 1990; Salazar y Rodríguez, 1996; Ayerbe et al., 1997) y por último, evolución en los tratamientos en relación a la interacción familiar (Ríos, 1984; Durán et al., 1994; Ochoa y Cebollada, 1996).

El objetivo de este estudio es doble, por un lado, conocer si hay diferencias en autoeficacia percibida en padres de dependientes a opiáceos según estén en grupos de tratamiento con metadona o en programas libres de drogas. Y por otro, conocer si hay diferencias en autoeficacia percibida entre los padres y las madres en ambos grupos.

\section{METOdOLOGÍA}

\section{SUJETOS}

Participaron 86 padres y 92 madres de dependientes a opiáceos de los cuales 40 padres y 44 madres pertenecían al grupo de metadona y 46 padres y 48 madres lo eran del grupo libre de drogas.

Los padres del grupo de metadona (grupo 1) tenían una media de edad de 61.1 años, un rango de 23, una edad mínima de 50 y una edad máxima de 73.I años. Las madres tenían una media de edad de 61.3 años, 
un rango de 30, una edad mínima de 47 y una edad máxima de 77 años. En el grupo libre de drogas (grupo 2) los padres tenían una media de edad de 60.5 años, un rango de 24, una edad mínima de 47 y una edad máxima de 71 años. Las madres tenían una media de edad de 61 años, un rango de 23, una edad mínima de 45 y una edad máxima de 68 años.

La profesión de los padres del grupo1 era de empleado o peón para el $26.9 \%$, jubilado para el $15.4 \%$ y otras diversas (empresario, administrativo, agricultor...) para el 53.8\%. Las madres eran jubiladas el $7.8 \%$, el $38 \%$ eran amas de casa y ejercían profesiones diversas (limpiadora, vendedora, auxiliar de clínica...) el 53.7\%. En el grupo libre de drogas, la profesión de los padres era de empleado o peón para el $22.2 \%$, jubilado para el $18.5 \%$ y otras diversas (mecánico, conductor, albañil, funcionario...) para el $51.9 \%$. Las madres eran jubiladas el 3.7\%, el 33.3\% eran amas de casa y ejercían profesiones diversas (limpiadora, empresaria, A.T.S....) el 62.9\%.

En cuanto al nivel de educación, los padres del grupo de metadona habían finalizado EGB o tenían estudios primarios el $78.4 \%$, eran analfabetos o leían con dificultad el $11.5 \%$, Bachillerato el $6.9 \%$ y otros el $3.2 \%$. Las madres habían finalizado EGB el $26.9 \%$, eran analfabetas el $7.6 \%$, tenían EGB sin concluir el $60.5 \%$, Bachillerato el $3.8 \%$ y otros el $1.2 \%$ En el grupo libre de drogas, los padres habían finalizado EGB o tenían estudios primarios el $79.6 \%$, eran analfabetos o leían con dificultad el 11.2\%, Bachillerato el $3.7 \%$ y otros el $5.4 \%$. Las madres habían finalizado EGB o estudios primarios el $62 \%$, eran analfabetas el $3.7 \%$, tenían EGB sin concluir $14.1 \%$, Bachillerato el $16.2 \%$ y otros el $4 \%$.

Participaron en el estudio 106 sujetos diagnosticados como dependientes a opiáceos (criterios DSM-IV) de una población de 450 personas en tratamiento en el Centro Provincial de Drogodependencias (C.P.D.) de Jaén.

El grupo 1, estuvo compuesto por 52 sujetos incluidos en un programa de mantenimiento con metadona (PMM) desde hacía un año o más. La muestra de este grupo se seleccionó al azar de una población de 200. La intervención asistencial en este grupo (revisiones mensuales) estaba orientada a lograr la estabilización a través de la sustancia y a los aspectos de educación para la salud o reducción de daños. El grupo 2 estuvo formado por 54 sujetos incluidos en programas libres de drogas que cumplían el criterio de 1 año de abstinencia o más, 43 de ellos mantenidos con antagonistas opiáceos (Naltrexona). Los sujetos de este grupo estaban incluidos en programas de intervención psicoterapéutica individual y/o familiar. Para la formación de este grupo se seleccionaron todos los sujetos en tratamiento que cumplían dicho criterio.

\section{MEDIDAS}

Las medidas utilizadas en este estudio fueron:

\section{Escala de Autoeficacia de Sherer y Adams (1982)}

Esta escala fue desarrollada para analizar las creencias que tienen los sujetos acerca de sus habilidades para manejar determinadas situaciones. Traducida y adaptada por Godoy (1990). Consta de 23 items, divididos en 2 subescalas. En la subescala de Autoeficacia General, el sujeto describe cómo maneja determinadas situaciones relacionadas con tareas personales. En la subescala de Autoeficacia Social, el sujeto describe cómo maneja determinadas situaciones sociales. En ambas escalas el sujeto debe indicar el grado de acuerdo de la frase que le describe o caracteriza en una escala de 1 a 5 , donde 1 significa estar fuertemente en desacuerdo y 5 fuertemente de acuerdo. Sherer y Adams (1982) señalan una consistencia interna, con el coeficiente alpha de Cronbach (1951), de .86 para la subescala de Autoeficacia General y de .71 para la subescala de Autoeficacia Social. Con respecto a la validez, los autores señalan una validez convergente con otras medidas características de personalidad, relacionadas con la eficacia general sin ser sinónimas. Encontraron, con el Cuestionario Locus de Control, una correlación significativa de -.173 con la escala de Control Personal y de -.132 para la subescala de Autoeficacia Social. Para la subescala de Autoeficacia general, se encontraron correlaciones negativas de - .51 con la Escala de Autoestima y de .43 con la Escala de Deseabilidad Social.

\section{Escala de Autoeficacia de Bäbler et al. (1993)}

Esta escala fue desarrollada primero con una versión de 20 items y después se redujo a una versión de 10 items (Jerusalem y Schwarzer, 1986, 1992; Schwarzer y Jerusalem, 1989). Se describen situaciones en las que se mide la propia capacidad del sujeto para manejar ciertos acontecimientos estresantes de la vida. La escala contiene una serie de frases. El sujeto debe indicar el grado en que la frase le describe 0 caracteriza en una escala de 1 a 4 , donde 1 significa incorrecto y 4 correcto. Bäbler et al. (1993) señalan una consistencia interna, con el coeficiente alpha de .74 a .92 según sea la muestra hombres, mujeres o emigrantes. Con respecto a la validez, los autores señalan una validez convergente con otras medidas características de autoeficacia, ansiedad, optimismo, depresión, etc. Encontraron, con el Cuestionario STAI (Laux et al. 1981) una correlación significativa de $r=-$ .54, con el test de Reacción (Saranson, 1984) una correlación de $r=-.42$, con la escala de Cheek y Buss (1981) una correlación de $r=-.58$, con el Inventario de Personalidad STPI (Schwarzer y Schwarzer, 1982, 
1983) una correlación $r=.44$, y una correlación con el IPC (Krampen, 1981) de $r=.40)$.

\section{PROCEDIMIENTO}

Para la cumplimentación de las medidas se les explicó a los padres que el objeto de las mismas era la obtención de un conocimiento sistematizado de la dinámica familiar con un fin terapéutico, los datos obtenidos servirían para poder ayudar más eficazmente a la familia durante el proceso de resolución del trastorno adictivo. La cumplimentación de las escalas tuvo lugar en las dependencias del Centro Provincial de Drogodependencias de Jaén y en los Centros Comarcales de Linares y Úbeda en horario de consulta (de 8 a 15 h).

\section{RESULTADOS}

Se realizó un análisis discriminante utilizando como variable de clasificación la variable grupo [Grupo metadona (1), grupo libre de drogas (2)] y como variables clasificatorias las puntuaciones de las Escalas de Autoeficacia (GSE y SSE) (Sherer y Adams, 1982) y la Escala de Autoeficacia de Bäbler et al. (1993). Para el proceso de selección de variables, se ha empleado el método paso a paso ("stepwise") con un criterio F de selección ( "to enter") de 3.00 y una F de exclusión ("to remove") de 2.95.

Los resultados indican que tres variables fueron incluidas en la función discriminante, las puntuaciones de los padres (varones) en la Escala de Autoeficacia de Bäbler et al. (1993), en las Escalas de Autoeficacia General y en la de Autoeficacia Social de Sherer y Adams (1982) siendo las que mejor discriminan a los sujetos en los grupos establecidos (véase la tabla 1).

La función de clasificación y los coeficientes de las variables para cada grupo se muestran en la tabla 1.

Utilizando la función de clasificación, se consiguió clasificar correctamente el $95 \%$ de los sujetos del grupo 1 y el $95.65 \%$ de los sujetos del grupo 2 resultando una clasificación global correcta de $95.35 \%$.

Los resultados de las escalas señalan que los padres del grupo libre de drogas puntúan más alto que los padres del grupo 1. Las puntuaciones medias de los sujetos en los items clasificatorios se muestran en la tabla 1.

Con el fin de comparar las puntuaciones de los padres y las de las madres se realizaron seis ANOVAS entre grupos con la variable parentesco como factor (padres, madres) y las variables Auto-eficacia como variables dependientes, tres para cada grupo.

Los resultados de las ANOVAS en el grupo de metadona indicaron una puntuación significativamente superior de las madres en la escalas de Bäbler: F(1,

TABLA 1: Presentación de los resultados del análisis de la función discriminante, función de clasificación y puntuaciones medias de los sujetos

\begin{tabular}{|c|c|c|c|c|c|}
\hline \multicolumn{6}{|c|}{$\begin{array}{l}\text { Análisis de la función discriminante } \\
\text { Wilks' Lambda: } 25018 \text { approx. } F(3,76)=75.927 p<.0000\end{array}$} \\
\hline SUBESCALAS & Coeficientes estandarizados & Lambda de Wilks & F-remove & p-level & R2 \\
\hline GSE padre & 1.73 & 0.57 & 96.78 & $3.4 \mathrm{E}-15$ & 0.75 \\
\hline SSE padre & -1.88 & 0.38 & 38.97 & 2.3E-08 & 0.87 \\
\hline Bäbler padre & 1.10 & 0.35 & 29.90 & 5.6E-07 & 0.69 \\
\hline \multicolumn{6}{|c|}{ Función de clasificación de las variables discriminantes para los grupos } \\
\hline SUBESCALAS & Grupo 1 & Grupo 2 & & & \\
\hline GSE padre & 3.92 & 4.90 & & & \\
\hline SSE padre & -5.94 & -7.39 & & & \\
\hline Bäbler padre & 2.62 & 3.38 & & & \\
\hline Constante & -80.86 & -129.29 & & & \\
\hline \multicolumn{6}{|c|}{ Puntuaciones medias de los sujetos por grupos } \\
\hline & Grupo 1 & Grupo 2 & & & \\
\hline SUBESCALAS & Hombres & Mujeres & Hombres & Mujeres & \\
\hline Bäbler & 21.8 & 31.8 & 30 & 36.5 & \\
\hline GSE (Sherer) & 51.3 & 62.4 & 65.7 & 66.5 & \\
\hline SSE (Sherer) & 16.3 & 21 & 22.4 & 21.6 & \\
\hline
\end{tabular}


82)=266.663; $p<.000$, una puntuación significativamente superior de las madres en la escala General de Sherer y Adams(GSE): $F(1,82)=373.816 ; p<.000$ y una puntuación significativamente superior de las madres en la escala Social de Sherer y Adams (SSE): F(1, 82) $=111.494 ; p<.000$.

Los resultados de las ANOVAS en el grupo libre de drogas indicaron una puntuación significativamente superior de las madres en la escalas de Bäbler: $F(1$, 92) $=19.191 ; p<.000$

Las puntuaciones medias de los sujetos por grupos se muestran en la tabla 1.

\section{DISCUSIÓN}

Los resultados del análisis discriminante señalan una puntuación más elevada en autoeficacia en las escalas de Bäbler y Sherer y Adams en los padres del grupo libre de drogas. No se han encontrado resultados significativos en las puntuaciones de autoeficacia de las madres. Considerando los grupos por separado, las madres puntúan más alto que sus maridos (en el grupo de metadona lo hacen en las escalas de Bäbler y Sherer y Adams, y en el grupo libre de drogas lo hacen en la escala de Bäbler). Ver Tabla 1. La puntuación más alta de los padres en el grupo libre de drogas se puede atribuir a que ya previamente al inicio del tratamiento eran más autoeficaces o bien al efecto del tratamiento.

Estos resultados aconsejan buscar estrategias psicoterapéuticas que tengan como meta mejorar la autoeficacia de los padres por los efectos beneficiosos en los hijos. Estas recomendaciones se avalan con las investigaciones llevadas a cabo en otros campos distintos al de las adicciones. Así, se ha señalado que los progenitores que poseen un nivel alto de autoeficacia favorecen la competencia de los hijos (Teti y Gelfand, 1991). De igual modo Cutrona y Troutman (1986) y Oliof y Aboud (1991) han referido que la autoeficacia parental es un factor protector contra la tensión emocional y el abatimiento. Schneewind (1999) en su revisión refiere que la autoeficacia de los padres se relaciona con una mayor orientación al control interno y a una dependencia menor de los controles externos en los hijos, así como con el modelado en adolescentes.

Así mismo, los resultados hallados nos hacen aconsejar la inclusión del padre en los procesos y programas de rehabilitación de los drogodependendientes. Para este trabajo clínico nos podemos orientar con las líneas que proponen Ríos (1984) y Araque (1999). Es decir en un contexto de trabajo familiar donde se tengan como objetivos la comunicación, la estructura, la solución de problemas y la participación en actividades externas de la comunidad. Esta inclusión del padre debería estar indicada tanto en los programas con sustitutivos como en los libres de drogas.
La madre en este trabajo aparece con mayor nivel de autoeficacia. Estos resultados son congruentes con los referidos en la literatura de las adicciones (Rios, 1984; Rodríguez y Sanz, 1989; Charro y Martínez, 1995).

Las diferencias entre los grupos en autoeficacia percibida, probablemente se relacionen con la percepción común, compartida tanto por las familias como por muchos profesionales, de que los programas sustitutivos con opiáceos no constituyen solución al problema sino que por el contrario favorecen el mantenimiento de éste y postergan su solución. De este modo este tipo de programas se asocia a sentimientos de ineficacia y fracaso. De forma opuesta, las familias implicadas en programas libres de drogas perciben mayor autoeficacia. Esta percepción posiblemente se relacione con sentimientos positivos y de esperanza en cuanto el probable éxito en la solución del problema adictivo del hijo.

En este punto parece necesario resaltar la importancia de la información transmitida acerca de los programas de sustitución y de sus objetivos por parte de los equipos terapéuticos. En este sentido cabe mencionar que el tratamiento con metadona constituye no sólo una estrategia de reducción de daños si no también un instrumento terapéutico para lograr el objetivo a medio-largo plazo de la abstinencia a opioides. De este modo los programas sustitutivos también están orientados al cambio cuando forman parte de un conjunto amplio de estrategias terapéuticas de tipo psicológico y social que incluye la asistencia a la familia.

Por otra parte, el trabajo con la autoeficacia de los padres podrían influir de forma beneficiosa en mejorar el clima familiar de los adictos, meta deseable y necesaria en los programas de rehabilitación. Se apoya esta recomendación con los resultados referidos por Scheewind $(1989,1999)$ con otras poblaciones que informa de correlaciones fuertes entre autoeficacia y clima familiar.

Estas orientaciones serían igualmente útiles para llevarlas a cabo en los programas preventivos. Se trataría de incorporar en los talleres de formación de las escuelas de padres orientación y entrenamiento en habilidades de solución de problemas, manejo de desacuerdos y otras funciones parentales para mejorar la autoeficacia. Así mismo, la eficacia conyugal podría ser un factor protector en el manejo de acontecimientos estresantes que acompañan a la adicción, al igual que en otro tipo de acontecimientos estresantes (Lazarus y Folkman, 1987; Bandura, 1999; Schneewind, 1999).

\section{REFERENCIAS}

Alameda, J., Araque, F., Canca, J., Rodríguez, M. y Rodríguez, S. (1987). Drogas ilegales en la ciudad de Jaén: Un estudio descriptivo. Jaén: Ayuntamiento de Jaén. 
Annis, M., Helen, Ph.D. (1990). Releapse to substance abuse: empirical findings within a cognitive-social learning approach. Journal of Psychoactive Drugs, vol 22 (2).

APA (1994). Diagnostic and statistical manual of mental disorders, $4^{\text {a }}$ edicción (DSM-IV). Washington, D.C.: APA.

Ayerbe, A., Espina, A., Pumar, B., García, E. y Santos, A. (1997). Clima familiar y características sociodemográficas en familias de toxicómanos. Adicciones, 3, 375390.

Bandura, A. (1977). Self-efficacy: toward a unyfyng theory of behabioral change. Psychological Review, 84, 191215.

Bandura, A. (1986). Social foundations of thoughtt and action. A social Cognitive Theory. New Jersey: Prentice Hall. (Trad. cast.: Pensamiento y acción: fundamentos sociales. Barcelona: Manínez Roca, 1987.

Bandura, A. (1988). Perceived self-efficacy: Exercise of control through self-belief. In J. P. Dauwalder, M. Perrez, y $\checkmark$. Hobi (Eds.), Annual series of European research in behavior therapy, 2, 27-59. Arnsterdarn/Lisse: Swets \& Zeitlinger.

Bandura, A. (1992a). Exercise of personal agency through the self-efficacy mechanism. In R. Schwarzer (Ed.), Self-efficacy Thought control of action (pp. 3-38). Washington, DC: Hemisphere.

Bandura, A. (1992b). Self-efficaey mechanism in psychobiologic functioning. In R. Schwarzer (Ed.), Self-efficacy: Thought control of action (pp. 355-394). Washington, DC: Hemisphere.

Bandura, A. (1999). Ejercicio de la eficacia personal y colectiva en sociedades cambiantes. En A. Bandura (Eds), Cómo afrontamos los cambios de la sociedad actual. Bilbao: Desclée de Brouwer, (pp. 19-55).

Barnea, Z., Teichman, M. y Rahav, G. (1992). Personality, cognitive and interpersonal factors in adolescent substance use: A longitudinal test of an integrative model. Journal of Youth and Adolescence, 21, 187-201.

Barry, K.L. y Fleming, M.F. (1990). Family cohesion, expressiveness and conflict in alcoholic families. British Journal of Addiction, 85, 81-87.

Baumrind, D. (1991). The influence of parenting style on adolescent competence and substance use. Journal of Early Adolescence, 11, 56-95.

Becoña, E., Frojan, M.J. y Lista, M.J. (1989). Comparison between two self-efficacy scales in maintenance of smoking cessation. Psycological Reports, 62, 359-362.

Bekir, P., Mclellan, T., Childress, R. y Gariti, P. (1993). Role reversal in families of substance misusers: A transgeneracional phenomenon. The International Journal of the Addictions, 28, 613-630.

Brook, J.S. y Whiteman, M. (1983). Stages of drug use in adolescence: Personality, peer and family correlates. Developmental Psychology, 19, 269-277.

Cancrini, L. (1991). La psicoterapia: Gramática y sintaxis. Barcelona: Paidós.
Charro, B. y Martínez M.P. (1995). Dinámica personal y familiar de los toxicómanos. Madrid: Universidad Pontificia de Comillas.

Coletti, M. (1998). La terapia familiar con las familias de Ios tóxicodependientes. $V$ Encuentro Nacional sobre Drogodependencias y su Enfoque Comunitario. Cádiz: Diputación Provincial (pp. 507-519).

Condiotte, M.M. y Lichtenstein, E. (1981). Self-efficacy and relapse in smoking cessation progranis. Journal of Consulting and Clininal Psychology, 49. 648-658.

Cooney, N,L., Kopel, S.A., McCkeon, P. (1982). Controlled relapse training and self-efficacy in exsmokers. Comunicación presentada en The annual meeting of the American Psichological Association. Washington, D.C.

Cutrona, C. E., y Troutman, B. R. (1986). Social support, infant temperament, and parenting self-efficacy: A mediational model of postpartum depression. Child Development, 57, 1507-1518.

Diclemente, C.C. (1981). Seif-efflcacy and smoking cessation maintenance: A preliminary report. Cognitive Therapy and Research, 5, 175-187.

Dishion, T.J., Patterson, G.R. y Reid, J.R. (1988). Parent and peer factor associated with drug sampling in early adolescence: Implications for treatment. NIDA Research Monograph Series, 77, 69-93.

Duran, A., Benito, R., Bueno, F.J., Sanchis, B. y Prado, M.J. (1994). La familia en el tratamiento de las drogodependendencias: Un estudio sobre actitudes y relaciones. Revista Española de Drogodependencias, 19, 297310.

Ellison, Ph. Y Hays, R., (1991). Beliefs about resistance selfefficacy and drug prevalence: Do they affect drug use?. The International Journal of the Addictions, 25, 1353-1378.

Fernández, J.J., Diaz, J., González, M.P. y Bobes, J. (1994). Perfil de la población drogodependiente en un programa de mantenimiento con metadona en Asturias. Adicciones, 6, 61-72.

Grove, J. R. (1993). Attributional correlates of cessation selPefficacy among smokers. Addictive Behaviors, 18, 311-320.

Jimenez, J.L. y Revuelta, A. (1991). La familia del toxicómano: Un estudio comparativo. Adicciones, 3, 133-140.

Jonhson, V. y Pandina, R. (1991). Effects of the family environment on adolescent substance use, deliquency and coping styles. American Journal of Drug and Alcohol Abuse, 17, 71-88.

Jurich, A.P. y Polson, C.J. (1985). Family factors in the lives of drug users and abusers. Adolescence, 77, 143-159.

Kandel D.B. (1978). Longitudinal research on drug use: Empirical findings and meethodological issues. Washington, D.C.: Hemisphere.

Kandel, D.B. (1987). Processes of adolescent socialization by parents and peers. The International Journal the Addictions, 22, 319-342. 
Kandel, D.B. (1990). Parenting styles, drug use and children's adjustment in families of younf adults. Journal of Marriage and the Family, 52, 183-196.

Kandel, D.B. (1992). Progression to regular marijuana involvement, phenomelogy and risk factors for near-daily use. En. M Glantz y R. Pickens (Eds), Vulnerability to drug abuse. Wasington, D.C., American Psycological Association (pp. 211-253).

Kaufman, E. (1985). Substance abuse and Family Therapy. New York: Grune y Stratton.

Kornblint A. L. y Cortazzo I. (1988). Detección de familias vulnerables a la drogadicción y estrategias de intervención pertinentes. Revista Española de Drogodependencias, 13, 3, 193-285.

Kornblit, A.L. y Mendes, A.M. (1990). Recaídas en drogadicción: Factores de riesgo. Revista Española de Drogodependencias, 2, 97-111.

Kosten, T.R., Jalali, M.D, Steidi, MSW y Kleber, M.D. (1987). Relationship of marital structure and interactions to opiate abuse relapse. American Journal Drug Alcohol Abuse, 13, 387-399.

Kosten, T.R., Jalili, B., Hogan, I. y Kleber, H.D. (1983). Family denial as a prognostic factor in oppiate addict treatment outcome. The Journal of Nervous Mental Disease, 171, 611-617.

Lazarus, R. S. y Folkman, S. (1984). Stress, appraisal, and coping. New York: Springer.

Litt, M. D. (1988). SelPefficacy and perceived control: Cognitive mediators of pain tolerance. Journal of Personality and Social Psychology, 54, 149-160

Locke, E. A., y Latham, G. P. (1990). A theory of goal setting and task performance. Englewood Cliffs, $\mathrm{Nj}$ : Prentice-Hall.

Machado, M.J. y Giron, S. (1993). Perfil psicosocial y retención de heroinómanos en tratamiento ambulatorio. Adicciones, 5, 109-125.

Marlatt, G. A., Baer, J. S., y Quigley, L. A. (1995). Self-efficacy and alcohol and drug abuse. In $A$. Bandura (Ed.), Se1f-efficacy in changing socíeties (pp. 289-315). New York: Cambridge University Press.

Marlatt, G. y Gordon, J. (1985). Release Prevention: Maintenance Strategies in addictive change. New York: Guilford Press.

Marlatt, G.A. (1993). La prevención de recaídas en las conductas adictivas: Un enfoque de tratamiento cognitivoconductual. En M. Casas y M. Gossop (Eds.). Tratamientos psicológicos en drogodependencias: Recaídas y prevención de recaídas. Barcelona: Citran, (pp. 137-153).

Marlatt, G.A., Baer, J. S., Quigley, L.A.. (1999). Autoeficacia y conducta adictiva. En A. Bandura (Eds), Cómo afrontamos los cambios de la sociedad actual. Bilbao: Desclée de Brouwer, (pp. 245-267).

McAuley, E. (1991). Efficacy attributional, and affective responses to exercise participation. Journal of Sport and Exercise Psychology, 13, 382-393.
Moos R.H, Finney J.W. y Cronkite R.C, (1990). Alcoholisme treatment. Context, process and outcome. New York: Oxford University Press.

Nakamura, K., Takano, T. y Iguchi, T. (1991). Family involvement for improving the abstinence rate in the rehabilitation process of female alcoholics. The International Journal of the Addictions, 26, 1055-1064.

Navarro, J. y Prieto, G. (1992). Patrones de emancipación familiar: Un estudio preliminar. Cuadernos de Terapia Familiar, 20, 19-31.

Needle, R., Doherty, W., Lavee, Y. y Brown, P. (1988). Familial interpersonal and intrapersonal correlates of drug use: A longitudinal comparison of adolescemts in treatment, drug-using adolescents not in treatment and non drugusing adolescents. The International Journal of the Addictions, 23, 1211-1240.

O'Farrell, TJ. (1989). Marital and family therapy in alcoholism treatment. Journal Substance Abuse Treatment, 6 , 23-29.

Ochoa, A. y Cebollada, C. (1994). Implicación familiar como predictor en un tratamiento de mantenimiento con antagonistas opiáceos. Adicciones, 6, 51-60.

Olioff, M., y Aboud, F. E. (1991). Predicting postpartum. dysphoria in primiparous mothers: Roles of perceived parenting selPefficacy and self-esteem. Journal of Cognitive Psychotherapy, 5, 3-14.

Otero J.M., Miron, L. y Luengo, A. (1989). Influence of the family and peer group on the use of drug by adolescents. The International Journal of the Addictions, 24, 1065-1082.

Paris, S. G., \& Newman, R. S. (1990). Developmental aspects of self-regulated leaming. Educational Psychologist, 25, 87-102.

Penk, W. (1979). Perceived family environments amog eEthnic grroups of compulsive heroin Users. Addictive Behaviors, 4, 297-309.

Recio, J.L., Santos, C., Sanchez, M.A., Escamilla, J.P., Plaza, L.A. y Barahona, M.J. (1991). El papel de la familia, los compañeros y la escuela en el abuso adolescente de drogas. Madrid: Asamblea de Cruz Roja.

Rios, J.A. (1984). Orientación y terapia familiar. Bilbao: Grijelmo.

Ripple, C.H. y Luthar, S.S. (1996). Familial factors in illicit drug abuse: An interdisciplinary perspective. American Journal Drug Alcohol Abuse, 22, 147-172.

Rodríguez, M., y Sanz, M. (1987). La familia del toxicómano desde un abordaje sistémico. Comunidad y Drogas, Monografías, 1, 87-103.

Rudkin, L., Hagell, A., Elder, G. H., y Conger, R. (1992). Perceptions of community well-being and the desíre to move elsewhere. Unpublished manuscript, University of North Carolina at Chapel Hill.

Salazar, I. y Rodríguez, A. (1994).Conflicto y dinámica familiar. Comunicación Psiquiátrica. Anales Universitarios Españoles de Psiquiatría. XVIII, 217-239.

Salinas, RC., O'farrell, T.J., Jones, WC. y Cutter, H.S. (1991). Services for families of alcoholics: A national survey of 
veterans affairs treatment programs. Journal Studies Alcohol, 52, 541-546.

Schneewind, K. A. (1994). Personality development in the context of education and socialization. In K. A. Schneewind (Eds.), Psychology of education and socialization. Encyc1opedia of Psychology. Educational Psychology (Vol. 1, pp. 197-225). Góttingen, Germany: Hogrefe.

Schwarzer, R. (1992). SelPefficacy in the adoption and maintenance of health behaviors: Theoretical approaches and a new model. In R. Schwarzer (Ed.), Self-eflicacy: Thought control of action (pp. 217-243). Washington, DC: Hemisphere.

Scweitzer, R.D. y Lawton, P.A. (1989). Drug abusers' perceptions of their parents. British Journal of Addiction, 84, 309-314.

Shneewind, K. A. (1999). Impacto de los procesos familiares sobre las creencias de control. En A. Bandura (Eds), Cómo afrontamos los cambios de la sociedad actual. Bilbao: Desclée de Brouwer, (pp. 105-131).

Sitharthan, T. y Kavanagh, D. J (1990). Role of sel-efficacy in predicting outcomes from a programe for controlled drinking. Drug and Alcohol Dependence, 27, 87-94.
Stanton, MD y Tood, T.C. (1988). Terapia familiar del abuso y adicción a las drogas. Buenos Aires: Gedisa.

Tarter, R.E., Blackson, T., Martin, C., Loeber, R. y Moss, H.B. (1993). Characteristic and correlates of child discipline practices in substance abuse and normal families. American Journal of Addictions, 2, 18-25.

Teti, D. M., y Gelfand, D. M. (1991). Behavioral competence among mothers of infants in the first year: The mediational role of matemal self-efficacy. Child Development, 62, 918-929.

Villamarín, F. (1990). Auto-Eficacia y conductas relacionadas con la salud. Revista de Psicología de la Salud, 2, 4564.

Villamarín, F. (1994). Autoeficacia: investigaciones en Psicología de la Salud. Anuario de Psicología, 61, 9-18.

Wiedenfeld, S. A., ULeary, A., Bandura, A., Brown, S., Levine, S., \& Raska, K. (1990). Impact of perceived selPefficacy in coping with stressors on components of the inunune system. Journal of Personality and Social Psychology, 59, 1082-1094.

Wood, R. y Bandura, A. (1989). Social cognitive theory of organizational management. Academy of Management Review, 14,361-384. 